UDC 517.977.5:626.54

A.A. Omelchuk,

G.V. Rudakova, DEng, Assoc.Prof.,

O.V. Polivoda, $\mathrm{PhD}$

Kherson National Technical University, 24 Berislavske Highway, 73008 Kherson, Ukraine; e-mail: rudik5a@rambler.ru

\title{
OPTIMAL CONTROL OF THE VESSEL MOTION PROCESS AT THE BROADSIDE SLIP
}

А.А. Омельчук, Г.В. Рудакова, О.В. Поливода. Оптимальне керування процесом переміщення судна на поперечному сліпі. Під час механізованого спуску або підйому судна за допомогою поперечного сліпа головними задачами $\epsilon$ забезпечення рівномірного руху об'єкта (судна разом з судновізними візками) та гарантоване запобігання позаштатних ситуацій. Мета: Метою роботи є формування і розв'язок задачі оптимального керування суднопідйомним комплексом типу сліп для забезпечення рівномірного, узгодженого та поступового переміщення великогабаритного об'єкту (судна). Матеріали $\boldsymbol{i}$ методи: У статті розглянуто питання оптимізації оперативного керування суднопідйомним комплексом під час спуску або підйому судна на сліпі. Результати: Запропоновано здійснювати керування переміщенням великогабаритного об'єкта шляхом узгодження сил натягу тросів окремих електроприводів з урахуванням поточної ситуації. Отримано модель у просторі станів, що описує рух судна в процесі спускупідйому. Сформульовано задачу оптимального керування складною багатоприводною системою з функціоналом мети, який дозволяє мінімізувати відхилення значень параметрів руху від заданих. Обгрунтовано доцільність застосування методів адаптивного керування зі спостерігачем для забезпечення відповідної надійності функціонування суднопідйомного комплексу.

Ключові слова: суднопідйомний комплекс, керований спуск судна на воду, оптимальне керування, простір станів, математична модель, адаптивна система керування.

A.A. Omelchuk, G.V. Rudakova, O.V. Polivoda. Optimal control of the vessel motion process at the broadside slip. The main purpose during the mechanized launching or lifting of a vessel using broadside slip is the uniform velocity motion providing of the object (vessel with launching trolleys) and the guaranteed prevention of emergencies. Aim: The aim of this work is the formulation and optimal control solution of a slip type vessel-lifting complex for providing the uniform velocity, coordinated, gradual relocation of the large vessels. Materials and Methods: The paper studies the matters of real-time control optimization of vessel-lifting complex during launching or lifting the vessel. Results: It is proposed to control the relocation of large object by funicular forces matching of the steel wire ropes of different motorized drives with account of current situation. The model in state space is obtained. This model describes the vessel relocation during the launching/lifting process. The optimal control solution of motorized multidrive system with goal functional is formulated. This solution allows minimizing the divergence of movement parameter values from given ones. It is substantiated the advisability of the use of adaptive control methods with observer to provide the corresponding reliability of vessel-lifting complex functioning.

Keywords: vessel-lifting complex, optimal control, state space, mathematical model, adaptive control systems.

Introduction. The main purpose during the mechanized launching or lifting of a vessel using broadside slip is the uniform velocity motion providing of the object (vessel with launching trolleys) and the guaranteed prevention of emergencies. Introducing the information control system, with a continuous monitoring of motion parameters and external factors' changes allow an increase in the vessel elevation systems' safety and reliability when moving large-sized objects, due to a timely and efficient operational control.

While the vessel launching and lifting widely used are such multi-drive vessel-lifting complexes as the slip [1]. The vessel motion along the slip's rails should be carried out at a constant speed, without distortions, at that because of various random factors contributing there is a risk of abnormal and emergency modes arising [2,3]. Previously designed models used to determine the vessel-lifting complexes functional modes and parameters are static thus ignoring external random factors, such as equipment wear, damage and corrosion of structural components, weather conditions impact etc.

In real time, especially in the slip complex critical operation mode, it is impossible to calculate the optimal control using those existing models. Therefore, actual is the problem of developing a dynamic model for large-sized objects at the broadside slip movement process, such a model allowing to analyze the external factors impact and broadside slip parameters' random changes, as well as to identify the lift complex multi-drive system operation modes throughout the process of vessel launch-

DOI 10.15276/opu.3.47.2015.11

(C) 2015 The Authors. This is an open access article under the CC BY license (http://creativecommons.org/licenses/by/4.0/).

COMPUTER AND INFORMATION NETWORKS AND SYSTEMS. MANUFACTURING AUTOMATION 
ing and lifting. For the "vessel with launching trolleys" complex object motion control it is advisable to use the slip winches' electric drive effort changes. The condition of maintaining the uniform motion without vessel rotation effect can be embodied with a condition of moments and forces equality on both sides of the base trolley (mass center). Wherein, depending on the current position of vessel at the broadside slip, formulated are the corresponding strategies and signals controlling the motion of launching trolleys linked with the common object carried (the vessel hull) [4].

The aim of this work is the formulation and optimal control solution of a slip type vessel-lifting complex for providing the uniform velocity, coordinated, gradual relocation of the large vessels.

Materials and Methods. To build the distributed "vessel with launching trolleys" object model, we proceed to consider the forces acting on it while the object moves towards the $x$ axis (Fig. 1)

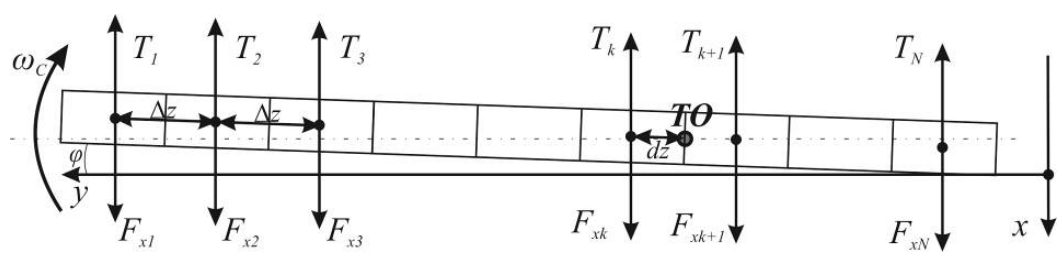

Fig. 1. Effects of forces at the "vessel with launching trolleys" object

The complex process of object's travel can be divided into two types of movement: translational motion characterized by velocity $v$ and acceleration $a$, and the rotational one characterized by the angular velocity $\omega$ and rotational angle $\varphi$.

The vessel translational motion equation has the following form

$$
\sum_{i=1}^{N} F_{x i}\left(l_{i}\right)-\sum_{i=1}^{N} T_{i}=m a
$$

where $T_{i}-i$-th trolley rope tension;

$F_{x i}\left(l_{i}\right)$ - projection of all forces influencing the $i$-th trolley and causing its motion at the broadside slip;

$l_{i}-i$-th trolley current location;

$m=m_{c}+N \cdot m_{\mathrm{T}}$ - mass of "vessel with launching trolleys" object,

$m_{c}$ - mass of the vessel,

$N$ number of trolleys,

$m_{\mathrm{T}}$ - mass of a trolley;

$a$ - acceleration of the "vessel with launching trolleys" object mass center.

The vessel rotational motion equation can be represented as

$$
J \frac{d \omega_{c}}{d t}=\sum M=-\sum_{i=1}^{N} F_{x i}[(k-i) \Delta z+d z] \cos \varphi+\sum_{i=1}^{N} T_{i}[(k-i) \Delta z+d z] \cos \varphi,
$$

where $J$ - vessel inertia moment,

$\omega_{c}$ - speed of the vessel rotation around the rotation point $(R P)$;

$\sum M$ - moment of forces applied to the object;

$\Delta z$ - distance between the neighboring trolleys' centers;

$d z$ - distance from rotation point (the object mass center) to the $k$-th trolley center (Fig. 1).

Based on the motion equations (1) and (2) we can write the model state equation for the "vessel with launching trolleys" object in the states space as follows 


$$
\begin{gathered}
\dot{x}_{1}=x_{2}, \quad \dot{x}_{2}=\frac{1}{m} \sum_{i=1}^{N} F_{x i}\left(x_{1}\right)-\frac{T_{m}}{m} \sum_{i=1}^{N} u_{i}, \\
\dot{x}_{3}=x_{4} \quad \dot{x}_{4}=\frac{1}{J} \sum_{i=1}^{N}\left(T_{m} u_{i}-F_{x i}\left(x_{1}\right)\right) \cdot[(k-i) \Delta z+d z] \cdot \cos x_{3},
\end{gathered}
$$

where $\quad x_{1}=l-$ vessel mass center motion along the $x$ axis;

$x_{2}=v-$ vessel mass center's translational motion speed along the $x$ axis;

$x_{3}=\varphi-$ vessel rotation angle;

$x_{4}=\omega-$ vessel rotation speed;

$\mathbf{u}=\left(\begin{array}{llll}u_{1} & u_{2} & \ldots & u_{N}\end{array}\right)^{T}$ - control vector, $u_{i}=T_{i} / T_{m}, i=\overline{1, N} ;$

$T_{m}$ - maximum allowable tension of the rope;

$F_{x i}\left(l_{i}\right)-i$-th drive's load caused by forces influencing the trolley at some point of trajectory $l_{i}$, value obtained with the expression [5]

$$
F_{x i}\left(l_{i}\right)=\left[\left(m_{\mathrm{T}}+m_{c}\right) g-\left(\frac{\rho g V_{T m}}{1+e^{-\delta_{24}\left(l_{i}-l_{24}\right)}}+\frac{\rho g V_{C m}}{1+e^{-\delta_{45}\left(l_{i}-l_{45}\right)}}\right)\right]\left[\sin \alpha-\left(\mu_{2}+\frac{\mu_{1}-\mu_{2}}{1+e^{\delta_{23}\left(l_{i}-l_{23}\right)}}\right) \cdot \cos \alpha\right] .
$$

The output model equations are formulated as

$$
y_{j}=x_{1}-[(k-j) \Delta z+d z] \sin x_{3}, j=\overline{1, r},
$$

where $r$ - number of outputs necessary to identify the complex object's motion parameters.

Given the small permissible rotation angles $\left(x_{3} \leq \varphi_{m}<2^{\circ}\right)$ we can assume $\sin x_{3} \approx x_{3}, \cos x_{3} \approx 1$, so the control object model (3) can be written in form

where

$$
\begin{gathered}
\dot{\vec{x}}=\vec{f}(\vec{x})+\mathbf{B}(k) \cdot \vec{u}, \\
\vec{y}=\mathbf{C}(k) \cdot \vec{x},
\end{gathered}
$$

$$
\begin{aligned}
& \vec{f}(\vec{x})=\left(\begin{array}{l}
x_{2} \\
\frac{1}{m} \sum_{i=1}^{N} F_{x i}\left(x_{1}-[(k-i) \Delta z+d z] x_{3}\right) \\
x_{4} \\
-\frac{1}{J} \sum_{i=1}^{N} F_{x i}\left(x_{1}-[(k-i) \Delta z+d z] x_{3}\right) \cdot[(k-i) \Delta z+d z]
\end{array}\right), \\
& \mathbf{B}(k)=\vec{b}\left(\begin{array}{cccc}
0 & 0 & \cdots & 0 \\
K_{1} & K_{1} & \cdots & K_{1} \\
0 & 0 & \cdots & 0 \\
K_{2}((k-1) \Delta z+d z) & K_{2}((k-2) \Delta z+d z) & \cdots & K_{2}((k-N) \Delta z+d z)
\end{array}\right) \text {, } \\
& \mathbf{C}(k)=\left(\begin{array}{cccc}
1 & 0 & (k-1) \Delta z+d z & 0 \\
1 & 0 & (k-N) \Delta z+d z & 0
\end{array}\right) \text { at } r=2, j_{1}=1, j_{2}=N .
\end{aligned}
$$

While forming the control matrix (8) the vector $\vec{b}=\left(\begin{array}{llll}b_{1} & b_{2} & \ldots & b_{N}\end{array}\right)$ is applied, whose components $b_{i}=1$ indicate the relevant drive's operating status and scale factors $K_{1}=-T_{m} / \mathrm{m}$, $K_{2}=T_{m} / J$. Elements of the matrix $\mathbf{C}$ correspond to the metering system structure.

The obtained model is nonlinear one, corresponding to the fourth order with variable structure (depending on equipment operated at a certain time).

The optimal control problem is formulated as the task of rotational motion stabilization (stopping) and maintaining the translational motion uniformity. In this regard, it is necessary to take into 
account the object movement parameters deviation from the preset values in the functional purpose. The functional purpose is formulated as:

$$
\begin{gathered}
\mathbf{J}=\int_{t_{0}}^{t_{f}}\left(\Delta \mathbf{x}^{T} \mathbf{R}_{1} \Delta \mathbf{x}+\Delta \dot{\mathbf{x}}^{T} \mathbf{R}_{2} \Delta \dot{\mathbf{x}}+\mathbf{u}^{T} \mathbf{Q u}\right) d t \rightarrow \min , \\
\Delta \mathbf{x}=\mathbf{x}-\mathbf{x}_{d}, \mathbf{x}_{d}=\left(\begin{array}{llll}
x_{1} & v_{d} & 0 & 0
\end{array}\right)^{T}, \Delta \dot{\mathbf{x}}=\left(\begin{array}{llll}
0 & \dot{x}_{2}-a_{d} & 0 & 0
\end{array}\right)^{T}
\end{gathered}
$$

At the following state limitations

$$
\begin{gathered}
0 \leq x_{3} \leq v_{m},\left|x_{3}\right| \leq \varphi_{m}, \\
0 \leq u_{i} \leq 1 \text { or } 0 \leq T_{i} \leq T_{m},
\end{gathered}
$$

and boundary conditions

$$
\mathbf{x}\left(t_{0}\right)=\mathbf{x}_{0} .
$$

The slip type vessel-lifting complex optimal control problem can be formulated as follows: for a given set of object equations (6), state limitations (11) and control limitations (12), with boundary conditions (13), required is to define a control mode $\mathbf{u}^{*}(t)$ and a phase trajectory $\mathbf{x}^{*}(t)$, at which the optimality criterion (10) will reach its minimum value.

The large-sized object (6) motion dynamics model is nonlinear, therefore to use the existing methods for control devices designing [6] the dynamic equations should be linearized around some steady state $\mathbf{x}_{s}=\left(x_{1 s}, \ldots, x_{4 s}\right)^{T}$ and the preset controls $\mathbf{u}_{s}=\left(u_{1 s}, \ldots, u_{N s}\right)^{T}$. So, taking into account $\mathbf{x}=\mathbf{x}-\mathbf{x}_{s}$ and $\mathbf{u}=\mathbf{u}-\mathbf{u}_{s}$, the object equation is expanded into Taylor's series, limited to the first order members. Because of these changes, the system (6) takes the form

$$
\begin{aligned}
& \frac{d x_{1}}{d t}=x_{2} \\
& \frac{d x_{2}}{d t}=\frac{1}{m} \sum_{i=1}^{N}\left[\left(\begin{array}{c}
\frac{\partial F_{x i}\left(x_{1 s}\right)}{\partial x_{1 s}} x_{1 s}+F_{x i}\left(x_{1 s}\right)+ \\
+((k-i) \Delta z+d z) x_{3 s} \frac{\partial F_{x i}\left(x_{1 s}\right)}{\partial x_{1 s}}
\end{array}\right) x_{1}+F_{x i}\left(x_{1 s}\right)((k-i) \Delta z+d z) x_{3}\right]+K_{1} \sum_{i=1}^{N} u_{i} \\
& \frac{d x_{3}}{d t}=x_{4}, \\
& \frac{d x_{4}}{d t}=K_{2} \sum_{i=1}^{N}\left[((k-i) \Delta z+d z)\left(\begin{array}{c}
\frac{\partial F_{x i}\left(x_{1 s}\right)}{\partial x_{1 s}} x_{1 s}+F_{x i}\left(x_{1 s}\right)+ \\
+((k-i) \Delta z+d z) x_{3 s} \frac{\partial F_{x i}\left(x_{1 s}\right)}{\partial x_{1 s}}
\end{array}\right) x_{1}+F_{x i}\left(x_{1 s}\right)((k-i) \Delta z+d z)^{2} x_{3}\right]+ \\
& +\sum_{i=1}^{N} \frac{T_{m}}{J}((k-i) \Delta z+d z) u_{i} .
\end{aligned}
$$

When determining the partial derivatives (14), we use the analytical dependence for the $i$-th drive load $F_{x i}$, which is obtained according to (4), that results in:

$$
\begin{aligned}
& \frac{\partial F_{x i}(l)}{\partial l}=\left(\rho g\left(\frac{V_{\mathrm{Tm}} \delta_{24} e^{-\delta_{24}\left(l-l_{24}\right)}}{\left(1+e^{-\delta_{24}\left(l-l_{24}\right)}\right)^{2}}+\frac{V_{c m} \delta_{45} e^{-\delta_{45}\left(l-l_{45}\right)}}{\left(1+e^{-\delta_{45}\left(l-l_{45}\right)}\right)^{2}}\right)\right)\left(\sin \alpha-\left(\mu_{2}+\frac{\mu_{1}-\mu_{2}}{1+e^{\delta_{23}\left(l-l_{23}\right)}}\right) \cos \alpha\right)+ \\
& +\left(\left(m_{\mathrm{T}}+m_{c}\right) g-\rho g\left(\frac{V_{\mathrm{Tm}}}{1+e^{-\delta_{24}\left(l-l_{24}\right)}}+\frac{V_{c m}}{1+e^{-\delta_{45}\left(l-l_{45}\right)}}\right)\right)\left(-\left(\mu_{1}-\mu_{2}\right) \cos \alpha \frac{\delta_{23} e^{\delta_{23}\left(l-l_{23}\right)}}{1+e^{\delta_{23}\left(l-l_{23}\right)}}\right) .
\end{aligned}
$$

Upon introducing (4) and (15) at (14) the linearized equations take the form 


$$
\begin{aligned}
& \frac{d x_{1}}{d t}=x_{2}, \\
& \frac{d x_{2}}{d t}=\frac{1}{m} \sum_{i=1}^{N}\left[\left[( x _ { 1 s } + ( ( k - i ) \Delta z + d z ) x _ { 3 s } ) \left[\rho g\left(\frac{V_{\mathrm{Tm}} \delta_{24} e^{-\delta_{24}\left(x_{1 s}-l_{24}\right)}}{\left(1+e^{-\delta_{24}\left(x_{1 s}-l_{24}\right)}\right)^{2}}+\frac{V_{c m} \delta_{45} e^{-\delta_{45}\left(x_{1 s}-l_{45}\right)}}{\left.\left(1+e^{-\delta_{45}\left(x_{1 s}-l_{45}\right.}\right)\right)^{2}}\right) \times\right.\right.\right. \\
& \times\left(\sin \alpha-\left(\mu_{2}+\frac{\mu_{1}-\mu_{2}}{1+e^{\delta_{23}\left(x_{1 s}-l_{23}\right)}}\right) \cos \alpha\right)+\left(-\left(\mu_{1}-\mu_{2}\right) \cos \alpha \frac{\delta_{23} e^{\delta_{23}\left(x_{1 s}-l_{23}\right)}}{1+e^{\delta_{23}\left(x_{1 s}-l_{23}\right)}}\right) \times \\
& \left.\times\left(\left(m_{\mathrm{T}}+m_{c}\right) g-\rho g\left(\frac{V_{\mathrm{T} m}}{1+e^{-\delta_{24}\left(x_{1 s}-l_{24}\right)}}+\frac{V_{c m}}{1+e^{-\delta_{45}\left(x_{1 s}-l_{45}\right)}}\right)\right)\right]+ \\
& +\left(\left(m_{\mathrm{T}}+m_{c}\right) g-\rho g\left(\frac{V_{\mathrm{Tm}}}{1+e^{-\delta_{24}\left(x_{1 s}-l_{24}\right)}}+\frac{V_{c m}}{1+e^{-\delta_{45}\left(x_{1 s}-l_{45}\right)}}\right)\right) \times \\
& \left.\times\left(\sin \alpha-\left(\mu_{2}+\frac{\mu_{1}-\mu_{2}}{1+e^{\delta_{23}\left(x_{1 s}-l_{23}\right)}}\right) \cos \alpha\right)\right] x_{1}+((k-i) \Delta z+d z) \times \\
& \times\left(\left(m_{\mathrm{T}}+m_{c}\right) g-\rho g\left(\frac{V_{\mathrm{Tm}}}{1+e^{-\delta_{24}\left(x_{1 s}-l_{24}\right)}}+\frac{V_{c m}}{1+e^{-\delta_{45}\left(x_{1 s}-l_{45}\right)}}\right)\right) \times \\
& \left.\times\left(\sin \alpha-\left(\mu_{2}+\frac{\mu_{1}-\mu_{2}}{1+e^{\delta_{23}\left(x_{1 s}-l_{23}\right)}}\right) \cos \alpha\right) x_{3}\right]+K_{1} \sum_{i=1}^{N} u_{i} \text {, } \\
& \frac{d x_{3}}{d t}=x_{4} \text {, } \\
& \frac{d x_{4}}{d t}=K_{2} \sum_{i=1}^{N}\left[\left[( x _ { 1 s } + ( ( k - i ) \Delta z + d z ) x _ { 3 s } ) \left[\rho g\left(\frac{V_{\mathrm{Tm}} \delta_{24} e^{-\delta_{24}\left(x_{1 s}-l_{24}\right)}}{\left(1+e^{-\delta_{24}\left(x_{1 s}-l_{24}\right)}\right)^{2}}+\frac{V_{c m} \delta_{45} e^{-\delta_{45}\left(x_{1 s}-l_{45}\right)}}{\left(1+e^{-\delta_{45}\left(x_{1 s}-l_{45}\right)}\right)^{2}}\right) \times\right.\right.\right. \\
& \times\left(\sin \alpha-\left(\mu_{2}+\frac{\mu_{1}-\mu_{2}}{1+e^{\delta_{23}\left(x_{1 s}-l_{23}\right)}}\right) \cos \alpha\right)+\left(-\left(\mu_{1}-\mu_{2}\right) \cos \alpha \frac{\delta_{23} e^{\delta_{23}\left(x_{1 s}-l_{23}\right)}}{1+e^{\delta_{23}\left(x_{1 s}-l_{23}\right)}}\right) \times \\
& \left.\times\left(\left(m_{\mathrm{T}}+m_{c}\right) g-\rho g\left(\frac{V_{\mathrm{Tm}}}{1+e^{-\delta_{24}\left(x_{1 s}-l_{24}\right)}}+\frac{V_{c m}}{1+e^{-\delta_{45}\left(x_{1 s}-l_{45}\right)}}\right)\right)\right]+ \\
& +\left(\left(m_{\mathrm{T}}+m_{c}\right) g-\rho g\left(\frac{V_{\mathrm{Tm}}}{1+e^{-\delta_{24}\left(x_{1 s}-l_{24}\right)}}+\frac{V_{c m}}{1+e^{-\delta_{45}\left(x_{1 s}-l_{45}\right)}}\right)\right) \times \\
& \left.\times\left(\sin \alpha-\left(\mu_{2}+\frac{\mu_{1}-\mu_{2}}{1+e^{\delta_{23}\left(x_{1 s}-l_{23}\right)}}\right) \cos \alpha\right)\right]((k-i) \Delta z+d z) x_{1}+((k-i) \Delta z+d z)^{2} \times \\
& \times\left(\left(m_{\mathrm{T}}+m_{c}\right) g-\rho g\left(\frac{V_{\mathrm{Tm}}}{1+e^{-\delta_{24}\left(x_{1 s}-l_{24}\right)}}+\frac{V_{c m}}{1+e^{-\delta_{45}\left(x_{1 s}-l_{45}\right)}}\right)\right) \times \\
& \left.\times\left(\sin \alpha-\left(\mu_{2}+\frac{\mu_{1}-\mu_{2}}{1+e^{\delta_{23}\left(x_{1 s}-l_{23}\right)}}\right) \cos \alpha\right) x_{3}\right]+\sum_{i=1}^{N} \frac{T_{m}}{J}((k-i) \Delta z+d z) u_{i} .
\end{aligned}
$$

The steady state vector $\mathbf{x}_{s}$ elements can be defined as the solution of equations system (16) with the zero left side.

The linearized equations in the states space can be written down in vector-matrix format as

$$
\mathbf{x}=\mathbf{A x}+\mathbf{B u},
$$

where the matrixes $\mathbf{A}$ and $\mathbf{B}$, having a permanent structure, change depending on the system functioning conditions and have the form 


$$
\begin{gathered}
\mathbf{A}=\left(\begin{array}{cccc}
0 & 1 & 0 & 0 \\
a_{21} & 0 & a_{23} & 0 \\
0 & 0 & 0 & 1 \\
a_{41} & 0 & a_{43} & 0
\end{array}\right), \\
\mathbf{B}=\left(\begin{array}{cccc}
0 & \cdots & 0 \\
0 & K_{1} & \cdots & K_{1} \\
K_{1} & 0 & \cdots & 0 \\
0 & K_{2}((k-2) \Delta z+d z) & \cdots & K_{2}((k-N) \Delta z+d z)
\end{array}\right),
\end{gathered}
$$

where the matrix $\mathbf{A}$ elements are found with the correlations

$$
\begin{aligned}
& a_{21}=\frac{1}{m} \sum_{i=1}^{N}\left[( x _ { 1 s } + ( ( k - i ) \Delta z + d z ) x _ { 3 s } ) \left[\rho g\left(\frac{V_{\mathrm{Tm}} \delta_{24} e^{-\delta_{24}\left(x_{1 s}-l_{24}\right)}}{\left(1+e^{-\delta_{24}\left(x_{1 s}-l_{24}\right)}\right)^{2}}+\frac{V_{c m} \delta_{45} e^{-\delta_{45}\left(x_{1 s}-l_{45}\right)}}{\left(1+e^{-\delta_{45}\left(x_{1 s}-l_{45}\right)}\right)^{2}}\right) \times\right.\right. \\
& \times\left(\sin \alpha-\left(\mu_{2}+\frac{\mu_{1}-\mu_{2}}{1+e^{\delta_{23}\left(x_{1 s}-l_{23}\right)}}\right) \cos \alpha\right)+\left(-\left(\mu_{1}-\mu_{2}\right) \cos \alpha \frac{\delta_{23} e^{\delta_{23}\left(x_{1 s}-l_{23}\right)}}{1+e^{\delta_{23}\left(x_{1 s}-l_{23}\right)}}\right) \times \\
& \left.\times\left(\left(m_{\mathrm{T}}+m_{c}\right) g-\rho g\left(\frac{V_{\mathrm{T} m}}{1+e^{-\delta_{24}\left(x_{1 s}-l_{24}\right)}}+\frac{V_{c m}}{1+e^{-\delta_{45}\left(x_{1 s}-l_{45}\right)}}\right)\right)\right]+ \\
& \left.+\left(\left(m_{\mathrm{T}}+m_{c}\right) g-\rho g\left(\frac{V_{\mathrm{Tm}}}{1+e^{-\delta_{24}\left(x_{1 s}-l_{24}\right)}}+\frac{V_{c m}}{1+e^{-\delta_{45}\left(x_{1 s}-l_{45}\right)}}\right)\right)\left(\sin \alpha-\left(\mu_{2}+\frac{\mu_{1}-\mu_{2}}{1+e^{\delta_{23}\left(x_{1 s}-l_{23}\right)}}\right) \cos \alpha\right)\right], \\
& a_{23}=\frac{1}{m} \sum_{i=1}^{N}((k-i) \Delta z+d z)\left(\left(m_{\mathrm{T}}+m_{c}\right) g-\rho g\left(V_{\mathrm{T} m} \frac{1}{1+e^{-\delta_{24}\left(x_{1 s}-l_{24}\right)}}+V_{c m} \frac{1}{1+e^{-\delta_{45}\left(x_{1 s}-l_{45}\right)}}\right)\right) \times \\
& \times\left(\sin \alpha-\left(\mu_{2}+\left(\mu_{1}-\mu_{2}\right) \frac{1}{1+e^{\delta_{23}\left(x_{15}-l_{23}\right)}}\right) \cos \alpha\right), \\
& a_{41}=K_{2} \sum_{i=1}^{N}\left[( x _ { 1 s } + ( ( k - i ) \Delta z + d z ) x _ { 3 s } ) \left[\rho g\left(\frac{V_{\mathrm{rm}} \delta_{24} e^{-\delta_{24}\left(x_{1 s}-l_{24}\right)}}{\left(1+e^{-\delta_{24}\left(x_{1 s}-l_{24}\right)}\right)^{2}}+\frac{V_{c m} \delta_{45} e^{-\delta_{45}\left(x_{1 s}-l_{45}\right)}}{\left(1+e^{-\delta_{45}\left(x_{1 s}-l_{45}\right)}\right)^{2}}\right) \times\right.\right. \\
& \times\left(\sin \alpha-\left(\mu_{2}+\frac{\mu_{1}-\mu_{2}}{1+e^{\delta_{23}\left(x_{1 s}-l_{23}\right)}}\right) \cos \alpha\right)+\left(-\left(\mu_{1}-\mu_{2}\right) \cos \alpha \frac{\delta_{23} e^{\delta_{23}\left(x_{1 s}-l_{23}\right)}}{1+e^{\delta_{23}\left(x_{1 s}-l_{23}\right)}}\right) \times \\
& \left.\times\left(\left(m_{\mathrm{T}}+m_{c}\right) g-\rho g\left(\frac{V_{\mathrm{Tm}}}{1+e^{-\delta_{24}\left(x_{1 s}-l_{24}\right)}}+\frac{V_{c m}}{1+e^{-\delta_{45}\left(x_{1 s}-l_{45}\right)}}\right)\right)\right]+ \\
& +\left(\left(m_{\mathrm{T}}+m_{c}\right) g-\rho g\left(\frac{V_{\mathrm{Tm}}}{1+e^{-\delta_{24}\left(x_{1 s}-l_{24}\right)}}+\frac{V_{c m}}{1+e^{-\delta_{45}\left(x_{1 s}-l_{45}\right)}}\right)\right) \times \\
& \left.\times\left(\sin \alpha-\left(\mu_{2}+\frac{\mu_{1}-\mu_{2}}{1+e^{\delta_{23}\left(x_{1 s}-l_{23}\right)}}\right) \cos \alpha\right)\right]((k-i) \Delta z+d z), \\
& a_{43}=K_{2} \sum_{i=1}^{N}((k-i) \Delta z+d z)^{2}\left(\left(m_{\mathrm{T}}+m_{c}\right) g-\rho g\left(\frac{V_{\mathrm{Tm}}}{1+e^{-\delta_{24}\left(x_{1 s}-l_{24}\right)}}+\frac{V_{c m}}{1+e^{-\delta_{45}\left(x_{1 s}-l_{45}\right)}}\right)\right) \times \\
& \times\left(\sin \alpha-\left(\mu_{2}+\frac{\mu_{1}-\mu_{2}}{1+e^{\delta_{23}\left(x_{15}-l_{23}\right)}}\right) \cos \alpha\right) \text {. }
\end{aligned}
$$

While analyzing the linearized system (17) controllability and observability the calculations were performed by the Kalman criterion [6]. Taking into account the structure of $\mathbf{A}$ and $\mathbf{B}$ matrixes system appearing as (18), the controllability matrix $\mathbf{L}$ has the form:

$$
\mathbf{L}=\left[\mathbf{B}|\mathbf{A B}| \mathbf{A}^{2} \mathbf{B} \mid \mathbf{A}^{3} \mathbf{B}\right],
$$




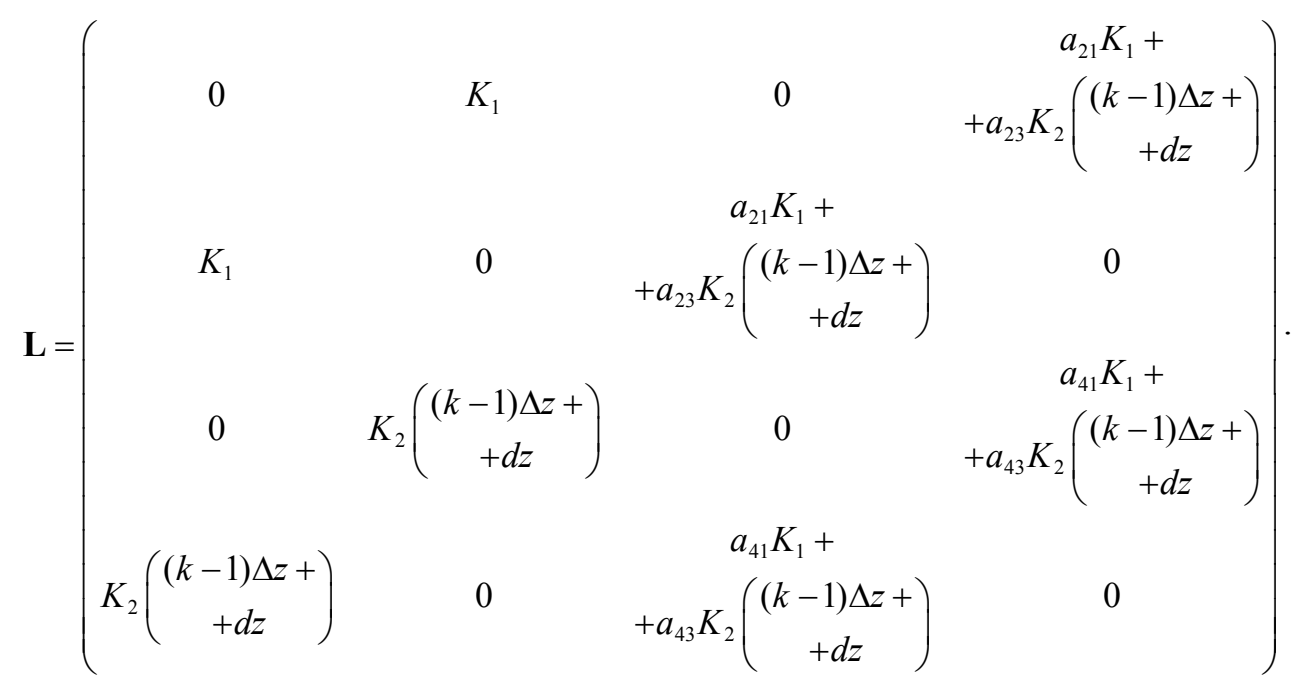

The matrix (21) determinant is defined as

$$
|\mathbf{L}|=\left(a_{41} K_{1}^{2}-a_{23} K_{2}^{2}((k-1) \Delta z+d z)^{2}-\left(a_{21}-a_{43}\right) K_{1} K_{2}((k-1) \Delta z+d z)\right)^{2} \neq 0,
$$

that indicates a complete controllability of an object, described with the model (6).

At forming the observability matrix $\mathbf{H}$ used is the matrix $\mathbf{C}$ (9) determined by the system output equations (5) that results in reducing matrix $\mathbf{H}$ to the following form:

$$
\mathbf{H}=\left[\mathbf{C}^{T}\left|\mathbf{A}^{T} \mathbf{C}^{T}\right|\left(\mathbf{A}^{T}\right)^{2} \mathbf{C}^{T} \mid\left(\mathbf{A}^{T}\right)^{3} \mathbf{C}^{T}\right]=\left[\begin{array}{cccccccc}
1 & 1 & 0 & 0 & h_{15} & h_{16} & 0 & 0 \\
0 & 0 & 1 & 1 & 0 & 0 & h_{27} & h_{28} \\
h_{31} & h_{32} & 0 & 0 & h_{35} & h_{36} & 0 & 0 \\
0 & 0 & h_{43} & h_{44} & 0 & 0 & h_{47} & h_{48}
\end{array}\right],
$$

where $\quad h_{31}=h_{43}=(k-1) \Delta z+d z, h_{32}=h_{44}=(k-N) \Delta z+d z, h_{15}=h_{27}=a_{21}+a_{41}[(k-1) \Delta z+d z]$,

$$
\begin{aligned}
& h_{16}=h_{28}=a_{21}+a_{41}[(k-N) \Delta z+d z], h_{35}=h_{47}=a_{23}+a_{43}[(k-1) \Delta z+d z], \\
& h_{36}=h_{48}=a_{23}+a_{43}[(k-N) \Delta z+d z] .
\end{aligned}
$$

The matrix (23) analysis confirms full observability of the system under consideration.

To identify large-sized object motion parameters that must be considered in the slip functioning mode control and drives control synthesis, the distance between some of object's points when movement from their original position, can be measured. General scheme to determine the two measurement points' motion parameters is shown in Fig. 2.

These measurements results serve in basis to determine the values and speed of the object mass center translational motion as well as the angle and speed of its rotational motion.

Taking into account the geometrical ratios, the rotation angles (at the angles small values) are found as

$$
x_{3}\left(t_{1}\right)=\varphi\left(t_{1}\right)=\frac{l_{2}\left(t_{1}\right)-l_{1}\left(t_{1}\right)}{z}
$$

and

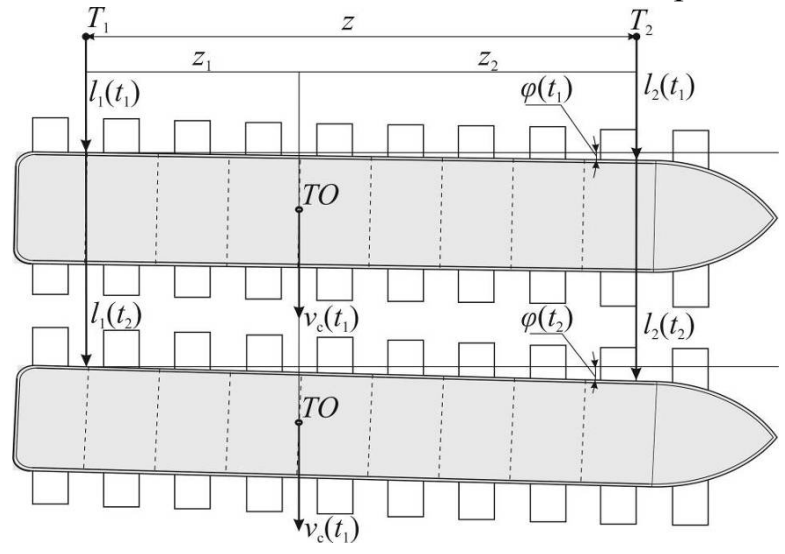

$$
x_{3}\left(t_{2}\right)=\varphi\left(t_{2}\right)=\frac{l_{2}\left(t_{2}\right)-l_{1}\left(t_{2}\right)}{z},
$$

Fig. 2. Scheme for the motion parameters determination 
where $l_{2}\left(t_{i}\right), l_{1}\left(t_{i}\right)$ - results of distance measurement at points $T_{2}$ and $T_{1}$ at the moment of time $t_{i}$, $z$ - distance between $T_{2}$ and $T_{1}$ points.

Using the known values (24) the rotation frequency can be defined as

$$
x_{4}\left(t_{2}\right)=\omega\left(t_{2}\right)=\frac{\varphi\left(t_{2}\right)-\varphi\left(t_{1}\right)}{\Delta t}, \Delta t=t_{2}-t_{1} .
$$

The complex object mass center speed is calculated directly from the expression

$$
x_{2}\left(t_{2}\right)=v_{c}\left(t_{2}\right)=\frac{l_{1}\left(t_{2}\right)-l_{1}\left(t_{1}\right)}{\Delta t}+z_{1} \cdot \omega\left(t_{2}\right), \Delta t=t_{2}-t_{1},
$$

where $z_{1}$ - distance from point $T_{1}$ to the complex object mass center (point of rotation).

On the basis of (26) the mass center $x_{c}=x_{1}$ motion value can be obtained as

$$
x_{1}\left(t_{2}\right)=v_{c}\left(t_{2}\right) \cdot \Delta t+x_{1}\left(t_{1}\right), \Delta t=t_{2}-t_{1} .
$$

To identify the "vessel with launching trolleys" complex object motion parameters, required is the preliminary calculation of vessel mass distribution over the slip trolleys and the complex object mass center to determine basic parameters (the basic trolley $k$ number and value $d z$ ).

As analyzed is the problem of maintaining an uniform and translational motion when vessel moving at the broadside slip after motors start, with trolleys acceleration and alignment, the initial conditions (at $t_{0}=0$ ) are selected as

$$
\mathbf{x}\left(t_{0}\right)=\mathbf{x}(0)=\left[x_{c}(0), v_{c}(0), \phi(0), \omega(0)\right]^{T}=\left[\begin{array}{llll}
0 & v_{d} & 0 & 0
\end{array}\right]^{T} .
$$

Taking into account the matrix $\mathbf{A}$ and vector $\mathbf{x}(0)$ structure, the identifiability matrix $\mathbf{W}$ structure is obtained in the following form

$$
\mathbf{W}=\left[\mathbf{x}(0)|\mathbf{A x}(0)| \mathbf{A}^{2} \mathbf{x}(0) \mid \mathbf{A}^{3} \mathbf{x}(0)\right]=\left[\begin{array}{cccc}
0 & v_{d} & 0 & a_{21} v_{d} \\
v_{d} & 0 & a_{21} v_{d} & 0 \\
0 & 0 & 0 & a_{41} v_{d} \\
0 & 0 & a_{41} v_{d} & 0
\end{array}\right] .
$$

The matrix determinant is $|\mathbf{W}|=-a_{41}^{2} \cdot v_{d}^{4} \neq 0$, thus the matrix rank $\operatorname{rang}(W)=4$ coincides with the system order. Therefore, an object described in the state space by the equations system (6) can be identified.

When solving the optimal control problem as to a moving complex object, represented by the model (17), it is required to achieve the functional purpose of a given state with the least possible losses of control resources. The quality of a non-accelerated motion process control (i.e., excluding the acceleration and braking segments) is determined by quadratic criterion appearing as

$$
\mathbf{J}=\int_{t_{0}}^{t_{f}}\left(\Delta \mathbf{x}^{T} \mathbf{R} \Delta \mathbf{x}+\mathbf{u}^{T} \mathbf{Q u}\right) d t,
$$

where the weight matrixes $\mathbf{R}$ and $\mathbf{Q}$ are symmetrical and positively identified.

For the criterion (30) an optimal regulator is set with the expression [6]

$$
\begin{gathered}
\mathbf{u}(t)=-\mathbf{F}(t) \Delta \mathbf{x}(t), \\
\mathbf{F}(t)=\mathbf{Q}^{-\mathbf{1}} \mathbf{B}^{\mathrm{T}} \mathbf{S}(t),
\end{gathered}
$$

where $\mathbf{S}(t)$ - symmetric and positively defined matrix, found from the Riccati equation

$$
\left(\dot{\mathbf{S}}+\mathbf{S A}+\mathbf{A}^{T} \mathbf{S}-\mathbf{S B Q}^{-1} \mathbf{B}^{T} \mathbf{S}+\mathbf{R}\right) \Delta \mathbf{x}(t)=0 .
$$


At constant matrixes $\mathbf{A}, \mathbf{B}, \mathbf{Q}, \mathbf{R}$ when $t \rightarrow \infty$, the $\mathbf{S}$ approaches to a stable value, which can be found by solving the Riccati algebraic matrix equation

$$
\mathbf{S B Q} \mathbf{Q}^{-1} \mathbf{B}^{T} \mathbf{S}-\mathbf{S A}-\mathbf{A}^{T} \mathbf{S}-\mathbf{R}=0 .
$$

For the vessel's motion control (lifting or launching processes) under conditions of changing values of the linearized model (19) matrix A components, depending onto the current object location at the slip and external factors, advisable is to use adaptive control methods. The matrix A components should be recalculated depending on the closest constant values, determined by the values of coordinate $x_{1}$, or identified using the recurrent algorithm. The state spaces object model matrixes $\mathbf{B}$ and $\mathbf{C}$ are stable, so their identification is not required.

The matrix A parameters setting recurrent algorithm is written as [7]

$$
\mathbf{A}(k+1)=\mathbf{A}(k)+\boldsymbol{\Gamma} \boldsymbol{\varepsilon}(k) \mathbf{y}^{T}(k),
$$

where $k$-calculation process step;

$\boldsymbol{\varepsilon}(k)=\mathbf{y}(k+1)-\mathbf{y}(k)-$ deviation of the object's and model's outputs;

$\boldsymbol{\Gamma}$ - weight coefficients matrix defined as

$$
\boldsymbol{\Gamma}(k)=\gamma(k) \mathbf{E}, \gamma(k)=\frac{\alpha}{k}, \alpha>0 .
$$

To form an optimal control with the expression (31) we must depart from the state vector variables measurement or evaluation, for which purpose can be used an optimal observer of the kind [6].

$$
\dot{\mathbf{x}}=[\mathbf{A}-\mathbf{K C}] \mathbf{x}+\mathbf{B u}+\mathbf{K y} .
$$

The observer synthesis consists of determining such coefficients matrix $\mathbf{K}$, for which the observer is asymptotically stable. For systems with constant parameters, the stability depends on the location of the matrix $\mathbf{A}-\mathbf{K C}$ characteristic numbers (the observer poles) in the complex plane.

The block diagram for an adaptive control system of complex object with observer and regulator, is shown in Fig. 3.

The parameters estimation module, identified at Fig. 3 as "PEM", does implement the recurrent identification algorithm of matrix A of the control object linearized model (LMCO). The matrix A components' values changes lead to the need for searching new values of observer matrix $\mathbf{K}$ and regulator coefficients $\mathbf{F}$ thus, accordingly, to get a new Riccati equation solution.

Results and Discussion. Estimated is that the broadside slip motion process control should be realized by funicular forces matching of the steel wire ropes of different motorized drives with account of current situation. A states-space nonlinear fourth order model, describing the linked object "vessel

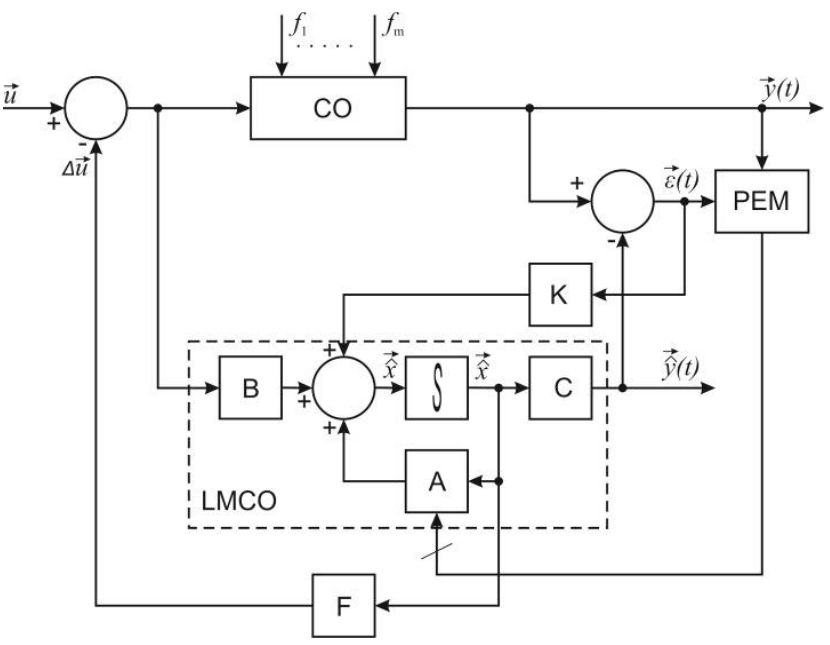

Fig. 3. Adaptive control system block diagram with launching trolleys" complex motion is obtained. The object fundamental properties model analysis confirmed the object's full controllability, observability and availability for its state identification through the measurements at two points.

The optimal control problem is formulated as the problem of rotational movement stabilization with uniform translational motion maintaining while accounting in the functional purpose the object parameters' deviations from preset values and the control costs. To control the vessels movement in the conditions of model parameters depending on the current object location at the slip and external 
factors, it is advisable to use the complex object adaptive control methods including the observer and the recurrent algorithm of the linearized model parameters identification.

The adaptive control system of vessel movement at the slip should be implemented as integral to the vessel-lifting complex computerized control system, to provide the slip electric drives' aligned operational control, aiming onto guaranteeing the appropriate reliability of the complex multi-drive system functioning thorough the vessel launching a lifting process.

\section{Література}

1. Rawson, K.J. Basic ship theory / K.J. Rawson, E.C. Tupper. - $5^{\text {th }}$ Ed. - Oxford: ButterworthHeinemann, 2005. $-727 \mathrm{p}$.

2. Омельчук, А.А. Проблеми узгодженого керування складною електромеханічною системою / А.А. Омельчук, Ю.О. Лебеденко, Г.В. Рудакова // Наукові праці Національного університету харчових технологій. - 2013. - № 49. - С. $19-23$.

3. Омельчук, А.А. Математическая модель электропривода слиповой тележки / А.А. Омельчук, А.В. Рудакова Ю.А. Лебеденко // Вестник ХНТУ. - 2014. - № 3(50). - С. 390 - 394.

4. Омельчук, А.А. Стратегия согласованного управления многоприводной системой спуска и подъема судна / А.А. Омельчук, А.В. Рудакова // Автоматика-2014: Матеріали 21-ї Міжнародної конференції з автоматичного управління, 23-27 вересня 2014 р., Київ. — К.: Вид-во НТУУ «КПІ», 2014. - С. $248-249$.

5. Омельчук, А.А. Моделирование пространственных параметров процесса спуска судна на воду с помощью слипа / А.А. Омельчук, А.В. Рудакова // Системні технології. — 2015. — № 4(99). С. $31-39$.

6. Ким, Д.П. Теория автоматического управления. Т. 2. Многомерные, нелинейные, оптимальные системы / Д.П. Ким. - М.: Физматлит, 2004. - 464 с.

7. Киричков, В.Н. Идентификация объектов систем управления технологическими процессами / В.Н. Киричков; под ред. А.А. Краснопрошина. - К.: Вища школа, 1990. — 264 с.

\section{References}

1. Rawson, K.J., \& Tupper, E.C. (2005). Basic Ship Theory ( $5^{\text {th }}$ Ed.). Oxford: Butterworth-Heinemann.

2. Omelchuk, A., Lebedenko, U., \& Rudakova, G. (2013). Problem of coordinate control of complex electromechanical system. Scientific Works of National University of Food Technology, 49, 19-23.

3. Omelchuk, A.A., Rudakova, G.V., \& Lebedenko, Y.A. (2014). The mathematical model of electric drive of slipway trolley. Bulletin of Kherson National Technical University, 3, 390-394.

4. Omelchuk, A.A., \& Rudakova, H.V. (2014). The coordinated control strategy of multidrive ship launching/lifting systems. In M.Yu. Ilchenko (Ed.), Proceedings of the XXI International Conference on Automatic Control (Automatics-2014) (pp. 248-249). Kyiv: NTUU “KPI”.

5. Omelchuk, A.A., Rudakova, H.V. (2015). Modelling the spatial parameters of ship launching process in a slipway. System Technologies, 4, 31-39.

6. Kim, D.P. (2004). Automatic Control Theory. Vol. 2, Dimensional, Nonlinear, Optimal and Adaptive Systems. Moscow: Fizmatlit.

7. Kirichkov, V.N. (1990). Identification of Objects in Control Systems for Engineering Processes. Kyiv: Vyshcha Shkola. 